\title{
Insomnia and Suicide Risk:
}

\section{A Multi-Study Replication and Extension among Military and High-Risk College Student Samples}

\author{
Raymond P. Tucker, PhD, Louisiana State University \\ Robert J. Cramer, PhD, UNC Charlotte \\ Jennifer Langhinrichsen-Rohling, PhD, UNC Charlotte \\ Ricky Rodriguez-Cue, UNC Charlotte \\ Susan Rasmussen, PhD, University of Strathclyde \\ Nicolas Oakey-Frost, BA, Louisiana State University \\ CAPT Michael Franks PsyD, MS, MsEd, MA, ABPP \\ Commander, US Public Health Service \\ Training Director, Naval Medical Center Psychology Training Programs \\ Navy Medicine Readiness and Training Command Portsmouth \\ Portsmouth, VA 23708 \\ CAPT Craig A. Cunningham, PhD, CNOR \\ Department Head, Nursing Research and Consultation Services \\ Navy Medicine Readiness and Training Command Portsmouth \\ Portsmouth, VA 23708
}

Corresponding Author: Robert J. Cramer, PhD, Associate Professor \& Belk Distinguished Scholar in Health Research, Department of Public Health Sciences, UNC Charlotte, 9201 University Blvd., Charlotte, NC, 28233, USA; (704) 687-6022; rcramer4@uncc.edu.

Data Availability Statement: Data are not publicly available due to United States Naval regulations.

Disclaimer: The views expressed in this article reflect the results of research conducted by the authors and do not necessarily reflect the official policy or position of the Department of the Navy, Department of Defense, or the United States Government. 
IRB Approval Statement and Funding Acknowledgement: The study protocol was approved by the Naval Medical Center Portsmouth Institutional Review Board in compliance with all applicable Federal regulations governing the protection of human subjects. Research data derived from an approved Naval Medical Center, Portsmouth, Virginia IRB, protocol; number NMCP.2017.0021. This work was supported by Navy Surgeon General Clinical Investigation Program funds.

Copyright Statement: Two of the authors are military service members. This work was prepared as part of their official duties. Title 17 U.S.C. 105 provides that "Copyright protection under this title is not available for any work of the United States Government." Title 17 U.S.C. 101 defines a United States Government work as a work prepared by a military service member or employee of the United States Government as part of that person's official duties.

\begin{abstract}
Objective/Background: A clear link between insomnia concerns and suicidal ideation has been shown in a variety of populations. These investigations failed to use a theoretical lens in understanding this relationship. Research within the veteran population has demonstrated that feelings of thwarted belongingness (TB), but not perceived burdensomeness (PB), mediate the insomnia and suicidal ideation relationship. Using two high risk samples, the present investigation replicated and extended this line of inquiry to include interpersonal hopelessness about TB, a key component of the Interpersonal Psychological Theory of Suicide.

Methods/Results/Conclusions: Using medical record review and survey data, study 1 replicated the finding that TB is a stronger explanatory factor of the insomnia to suicidal ideation/suicide risk relationship in a sample of $\mathrm{N}=200$ treatment-seeking active-duty personnel. Study 2 found that insomnia symptoms had an indirect effect on suicidal ideation through TB and PB but not interpersonal hopelessness in a sample of $\mathrm{N}=151$ college students with a history of suicidal
\end{abstract} thoughts and/or behaviors. TB was the only mediator of the insomnia-suicide attempt likelihood link and insomnia to clinically significant suicide risk screening status. Limitations include cross-sectional design of both studies and the lack of formal diagnoses of insomnia. Implications and future research directions are discussed.

Key words: Insomnia, Suicidal Ideation; Suicide; Military; Interpersonal Theory; Hopelessness 


\section{Introduction}

Insomnia is globally recognized as a major public health concern (Chattu et al., 2019). Insomnia is characterized by difficulties initiating and maintaining sleep, and dissatisfaction with both sleep quality and quantity (Kupfer \& Reynolds, 1997). Whilst the prevalence rates reported in different studies vary considerably depending on the definition and method used to assess insomnia (Bos \& Macedo, 2019), epidemiological studies generally estimate that around 5-10\%

of the general population is affected by the disorder (Ellis, Perlis, Neale, Espie \& Bastien, 2012; Perlis et al., 2020). Insomnia appears to be especially prevalent in some populations. For example, research with current and former military service members has indicated that up to $74 \%$ of this population meet diagnostic criteria for insomnia (Klingaman, Brownlow, Boland, Mosti, \& Gehrman, 2018). The findings underscore the consequences of insomnia which are wide-ranging and include increased mortality risk, reduced occupational functioning, reduced quality of life, poor physical and mental health (e.g. Bos \& Macedo, 2019).

One particularly concerning correlate of insomnia is increased risk for suicidal thoughts and behaviors (STBs; Park, Kwang, \& Kim, 2019; Woznica, Carney, Kuo, \& Moss, 2015). The relationship between insomnia and STBs has been demonstrated in the general population (Park et al., 2019), civilian medical patients receiving services at a sleep clinic (Krakow, Ribeiro, Ulibarri, Krakow, \& Joiner, 2011), active-duty military (Ribeiro et al., 2012), and military veterans (Bishop, Crean, Hoff, \& Pigeon, 2019; Britton, et al., 2019).

Recent empirical work has shed more light on the mechanisms that explain the link between insomnia and STBs. For example, in a survey of over 12,000 female adolescents, insufficient sleep maintained a direct association with suicidal ideation (SI), even after controlling for co-occurring symptoms of depression (Park et al., 2019). However, depressive 
symptoms were shown to partially mediate the relationship between insufficient sleep and increased risk for SI. In fact, depression has consistently been shown to mediate the relationship between insomnia and STBs (e.g., Bishop et al., 2019; Britton, et al., 2019; Woznica et al., 2015). However, in a separate study of medical patients accessing a sleep clinic, sleep quality continued to be associated with SI, even after controlling for depressive symptoms and daytime fatigue (Krakow et al., 2011). Likewise, in one young active-duty military sample, sleep problems were shown to be better cross-sectional and longitudinal predictors of STBs than either depressive symptoms or hopelessness (Ribeiro et al., 2012). Thus, while there is growing consensus about the link between insomnia and suicide, the understanding of the mechanisms underlying this link is rudimentary.

The ability to identify these mechanisms represents an important step toward understanding how STBs develop and are maintained. In addition, understanding these mechanisms may also offer opportunities to develop or utilize existing sleep and suicide intervention strategies like Cognitive Behavioral Therapy for Insomnia (CBT-I; Perlis et al., 2006). Although a number of potential explanations have been proposed (e.g., impaired emotional regulation; Bernert \& Joiner, 2007), a theoretical framework that explains the relationship may be particularly helpful. For example, Kleiman and Anestis (2015) advocated the use of theoretically informed models to examine the complexity of the pathway to STBs, whilst Woznica and colleagues (2015) argued specifically for a renewed research agenda to explore the mechanisms linking insomnia to suicide. The Interpersonal Psychological Theory of Suicide (IPTS; Joiner, 2007; Van Orden et al., 2010) offers one such framework to explain the association between insomnia, suicide risk factors, and STBs. The IPTS posits that active suicidal desire emerges as a consequence of an individual simultaneously experiencing thwarted 
belongingness (TB) and perceived burdensomeness (PB). TB is characterized by feelings of loneliness or a lack of meaningful social relationships and PB reflects an individual's belief that they are a liability to others, coupled with perceptions of self-hatred (Van Orden et al., 2010). Importantly, active suicidal desire is hypothesized to develop when TB and PB are seen as chronic, stable, or hopelessly intractable.

Systematic reviews and meta-analyses have demonstrated support for TB and PB as independently associated with SI across a number of different populations (Chu et al., 2017; Ma et al., 2016). For example, a recent systematic review of the psychological mechanisms that explain the relationship between sleep problems and STBs found that TB and PB were constructs with robust evidence for their explanatory role in the insomnia-SI pathway (Chu et al., 2017; Golding et al., 2015; Littlewood, Kyle, Pratt, Peters \& Gooding, 2017; Nadorff et al., 2014). Later investigations have demonstrated the particular relevance of TB in explaining the insomnia and SI link. For example, Chu et al (2017) found that TB significantly accounted for the association between insomnia and SI, cross-sectionally and longitudinally across diverse samples (students, psychiatric outpatients, primary care patients, and firefighters) which has been replicated in samples of veterans and active duty military service members (Britton et al., 2019; Hom et al., 2017). Thus, the IPTS may provide a useful framework for understanding the link between insomnia and suicidal risk. However, there appears to be a lack of research regarding the importance of hopelessness about these two constructs, as well as comparing differences in the strength of IPTS explanatory pathways.

Whereas hopelessness may be defined as negative expectations about the self and the future (Beck, Weissman, Lester, \& Trexler, 1974), the construct of interpersonal hopelessness is specific to negative expectations about the future resolution of $\mathrm{TB}$ and $\mathrm{PB}$ or that these states are 
hopelessly intractable (Tucker et al., 2018). For example, TB may manifest in thoughts such as, "no one in my life really cares about me" but this thought content does not include perceptions of hopelessness such as, "no one cares about me and no one ever will." Van Orden et al. (2010) proposed that interpersonal hopelessness is key in the development of active suicidal desire, a proposition that later garnered empirical support. For example, Tucker and colleagues (2018) found that the interaction of $\mathrm{TB}, \mathrm{PB}$, interpersonal hopelessness predicted SI above and beyond all possible two-way interactions of the foregoing risk factors, a finding not replicated when general hopelessness was considered instead. Additionally, Mandracchia and colleagues (2019) found that interpersonal hopelessness accounts for additional variance in SI above the effects of TB and PB. In light of this budding research, interpersonal hopelessness may be of relevance in explaining the insomnia and SI relationship. Indeed, insomnia may confer vulnerability for, or exacerbate extant dysphoric cognitions like, hopelessness as well as the links between these symptoms (i.e., TB and PB). Thus, an initial study regarding the potential explanatory role of interpersonal hopelessness between insomnia and SI is needed.

\section{The Present Studies:}

The literature above outlines the need for a better understanding of the link between insomnia, STBs, and potential mechanisms as outlined in the IPTS in (1), higher risk active duty military service members and young adults with lived suicide experience, and (2) that considers the role of interpersonal hopelessness. Research regarding how TB and PB relate to insomnia and SI have been performed in veteran only samples (Britton et al., 2019; Hom et al., 2017 study 3), mixed samples of veterans and active duty military service members (Hom et al., 2017 study 1), and Army recruiters (Hom et al., 2017 study 2). Currently, no research has investigated these relationships in active duty service members seeking mental health treatment, a limitation given 
the pervasive nature on insomnia in active duty service members (Klingman et al., 2018). Thus, Study 1 of the current manuscript investigates the relationship between insomnia symptoms, SI, and both TB and PB in a treatment-seeking sample of active duty military service members. It was hypothesized that TB but not PB would mediate the insomnia symptom to suicide risk relationship as demonstrated in previous research (Chu et al., 2017). This investigation parsed out varying elements of suicide risk as separate outcomes, including frequency of SI, one's own perceived risk for suicide attempt, and clinically significant suicide risk to better understand if effects are specific to one (or more) indicators of risk.

Although Study 1 expands upon the extant literature in its use of a clinical active duty military sample, it still, like all other previous investigations, fails to consider the role of interpersonal hopelessness. Thus, Study 2 investigated whether interpersonal hopelessness mediated the insomnia symptom to suicide risk relationship in a sample of college students with a history of STBs. Given the strong link between SI and interpersonal hopelessness even when other risk factors have been considered (Tucker et al., 2018), it was hypothesized that interpersonal hopelessness would mediate the insomnia symptom to suicide risk relationship independent of the effects of TB and PB alone.

\section{Study 1 Material and Methods}

\subsection{Participants.}

A total of 200 treatment-seeking active duty service members took part in this investigation. ${ }^{1}$ The sample was of young adult average age $\left(M_{\text {years }}=26.99, S D=6.41\right)$. Predominant demographic characteristics were male sex $(n=154,77 \%)$, White race $(n=125$,

\footnotetext{
${ }^{1}$ Data from this study were drawn from a larger investigation on active duty service member coping and mental health. Demographics for this sample are reported elsewhere in the literature (see Cunningham et al., 2020), but insomnia-focused analyses in the present investigation are completely unique from the prior work.
} 
$62.5 \%)$, and enlisted rank $(n=182,91.0 \%)$. A majority of the sample $(n=172,86.0 \%)$ were recruited from the substance abuse treatment program, whereas the remaining participants $(n=28,14.0 \%)$ were enrolled from the mental health "Access-to-Care" clinic. Table 1 contains descriptive statistics for the sample health related variables. Almost half of participants (48\%) qualified as elevated risk for suicide (Osman et al., 2001), whereas mean levels of insomnia were subthreshold (Morin et al., 2011).

\subsection{Measures}

2.2.1 Demographics. Demographic information such as age, sex, race, and rank were extracted from each participant's record in the Behavioral Health Data Portal (Department of the Navy, 2016), a standardized electronic database housing patient initial assessment and further health information.

2.2.2 Suicidal Thoughts and Behaviors (STBs). The Suicidal Behaviors QuestionnaireRevised (SBQ-R; Osman et al., 2001) is a flexible brief screener of lifetime STBs. It contains 4 items, each assessing a different aspect of suicidal behavior. These include lifetime ideation and attempt (item 1), 12-month ideation frequency (item 2), communication of intent (item 3), and likelihood of a future attempt (item 4). Items can be summed for a total score which consistently displays high internal consistency (e.g., Chu et al., 2017; Osman et al., 2001; Stanley et al., 2017). SBQ-R items have also been used as specific metrics of attempt, ideation and future behavior in prior literature (e.g., Lund et al., 2019; Winer et al., 2016). Within the present study, we employed items 2 and 4 to capture 12-month ideation and likelihood of future attempt, respectively. The SBQ-R also contains a recommended cut-score for non-inpatient samples; this score demarcates "low" versus "elevated" clinical suicide risk (Osman et al., 2001). The SBQ-R 
clinical cut scores have also been applied widely in prior studies (e.g., Cramer, 2017, 2019; Osman et al., 2001); as such, we employed it as an assessment of suicide risk.

2.2.3 Insomnia Symptoms. The Insomnia Severity Index (ISI; Morin, 2003, Morin et al., 2011) was used to assess insomnia symptoms. This 7 -item measure captures current insomnia severity ( 3 items), distress caused by insomnia ( 3 items, e.g., distress or dissatisfaction due to sleep disturbance), and inference with one's daily functioning (1 item). The ISI consistently demonstrates high internal consistency (e.g., Chu et al., 2017; DeShong \& Tucker, 2019; Morin et al., 2011). Internal consistency in the present sample was $\alpha=.91$.

2.2.4 Interpersonal Cognitions. The Interpersonal Needs Questionnaire (INQ; Van Orden et al., 2012) was used to assess interpersonal beliefs. This 15-item inventory contains two subscales: PB (9 items) and thwarted belonging (6 items). INQ subscales consistently demonstrate acceptable internal consistency (e.g., Chu et al., 2017; Van Orden et al., 2012). Internal consistency was high for both subscales in the present sample: $\mathrm{PB}(\alpha=.93)$ and $\mathrm{TB}(\alpha=$ $.93)$.

\subsection{Procedure}

This study was approved by Institutional Review Boards from the Military Treatment Facility (MTF) and one university partner. Data were drawn from a combination of medical record review via the Behavioral Health Data Portal (BHDP which includes demographics and the ISI; Department of the Navy, 2016) and a subsequent self-report questionnaire battery (including the INQ and SBQ-R). Participants were recruited from two clinics within a major MTF: outpatient mental health and substance abuse treatment. All participants were recruited, consented, and enrolled in the study prior to treatment. Specifically, a research coordinator shared study information (e.g., procedure, risks, benefits) both in writing and by oral 
communication with study-eligible active duty service members upon first contact in specified treatment clinics. Study eligibility included current active duty status, a minimum age of 18 years, and seeking treatment in one of the two study clinics. Consenting participants provided study participation agreement in writing providing the research coordinator permission to record de-identified demographic and health related information (e.g., insomnia scores, health history) into a master database. These data were joined by a subsequent self-report questionnaire battery (e.g. measures of STBs, TB/PB) completed by study participants. All study procedures were reviewed by the appropriate institutional review board.

\subsection{Analytical Strategy}

Missing data were replaced via multiple imputation (Enders, 2017). Pearson correlations assessed bivariate relationships among suicide related outcomes, insomnia, and interpersonal cognitions. Mediation analyses were conducted using SPSS PROCESS macros (Hayes, 2018). Specifically, PROCESS model 4 allows for inspection of multiple mediators (i.e., PB and TB) of the insomnia-suicide risk link. A total of three parallel mediation analyses were conducted, one with each of the following outcomes: (1) 12-month SI (SBQ-R item 2); (2) future likelihood of suicide attempt (SBQR-item 4), and (3) clinical suicide risk (application of cut-score yielding no versus elevated risk groups; Osman et al., 2001). These three outcomes were chosen as SI has been consistently linked to insomnia and TB/PB (Chu et al., 2017), patient perceived suicide risk is an important treatment target in suicide-specific interventions (Jobes, 2017), and the overall clinical risk cut-off of the SBQ-R is a meaningful indicator of risk for suicidal behavior at threemonth follow-up in current/former service members (Gutierrez et al., 2020). Five thousand bootstrapped resamples were used, with $95 \%$ bias corrected and accelerated confidence intervals (BCa CIs) to determine whether mediation is present. Age and gender were added as covariates 
for all three models given their relationship with suicidal thoughts (Crosby et al., 2011; Stanley et al., 2016).

\section{Study 1 Results}

Missing data on any scale items was low (maximum 3.5\%). Table 2 contains bivariate associations. Insomnia displayed significant small positive correlations with suicide related outcomes and interpersonal cognitions. Interpersonal cognitions displayed significant, positive, moderate, associations with suicide related outcomes.

\subsection{Parallel mediation findings}

The results of the full model significantly predicted past two-week $\mathrm{SI}\left(R^{2}=.35, F(5\right.$, $185)=19.90, p<.001$; Figure 1). The direct effect of insomnia symptoms on SI was not significant $(\beta=-.04,95 \% \mathrm{CI}[-.03, .02])$. An indirect effect for insomnia symptoms on SI was observed through $\mathrm{TB}(\beta=.04,95 \% \mathrm{CI}[.02, .06])$ and $\mathrm{PB}(\beta=.01,95 \% \mathrm{CI}[.01, .21])$ A pairwise comparison of the indirect effects demonstrated no a stronger effect for TB compared to $\mathrm{PB}(\beta=.11,95 \%$ $\mathrm{CI}[.01, .22])$ 
Figure 1.

Indirect Effect Analysis of Insomnia Symptoms on Suicide Ideation Through Thwarted Belongingness and Perceived Burdensomeness

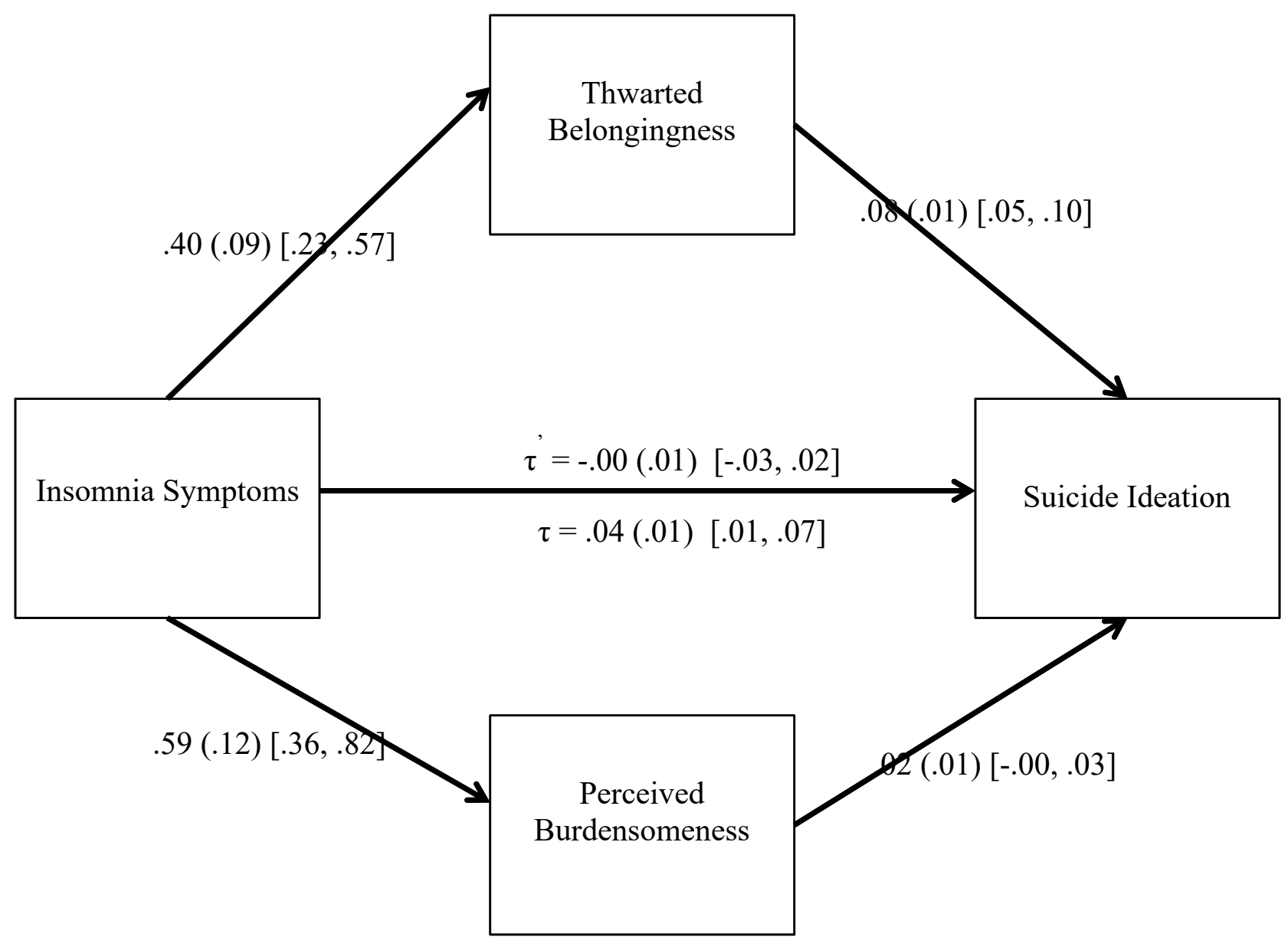

Note. Unstandardized regression coefficients, (standard errors), and [95\% confidence intervals] presented; $\tau^{\prime}=$ direct effects of independent variable on dependent variable; $\tau=$ total effects of independent variable on dependent variable; Suicide Ideation $=$ frequency of suicide ideation in the past year. 
The results of the full model significantly predicted future likelihood of a suicide attempt, $\left(R^{2}=.46, \mathrm{~F}(5,185)=52.91, p<.001\right.$; Figure 2$)$. The direct effect of insomnia symptoms on future likelihood of a suicide attempt was not significant $(\beta=-.01,95 \% \mathrm{CI}[-.03, .01])$. Insomnia evinced an indirect relationship with self-rated likelihood of a future suicide attempt through TB $(\beta=.03$, $95 \% \mathrm{CI}[.03, .07])$ and $\mathrm{PB}(\beta=.02,95 \% \mathrm{CI}=[.01, .03])$. A pairwise comparison of the indirect effects demonstrated no statistical difference in strength between the two effects $(\beta=.01,95 \%$ CI[-.01, .03]).

\section{Figure 2.}

Indirect Effect Analysis of Insomnia Symptoms on Perceived Risk for Future Suicide Attempt Through Thwarted Belongingness and Perceived Burdensomeness

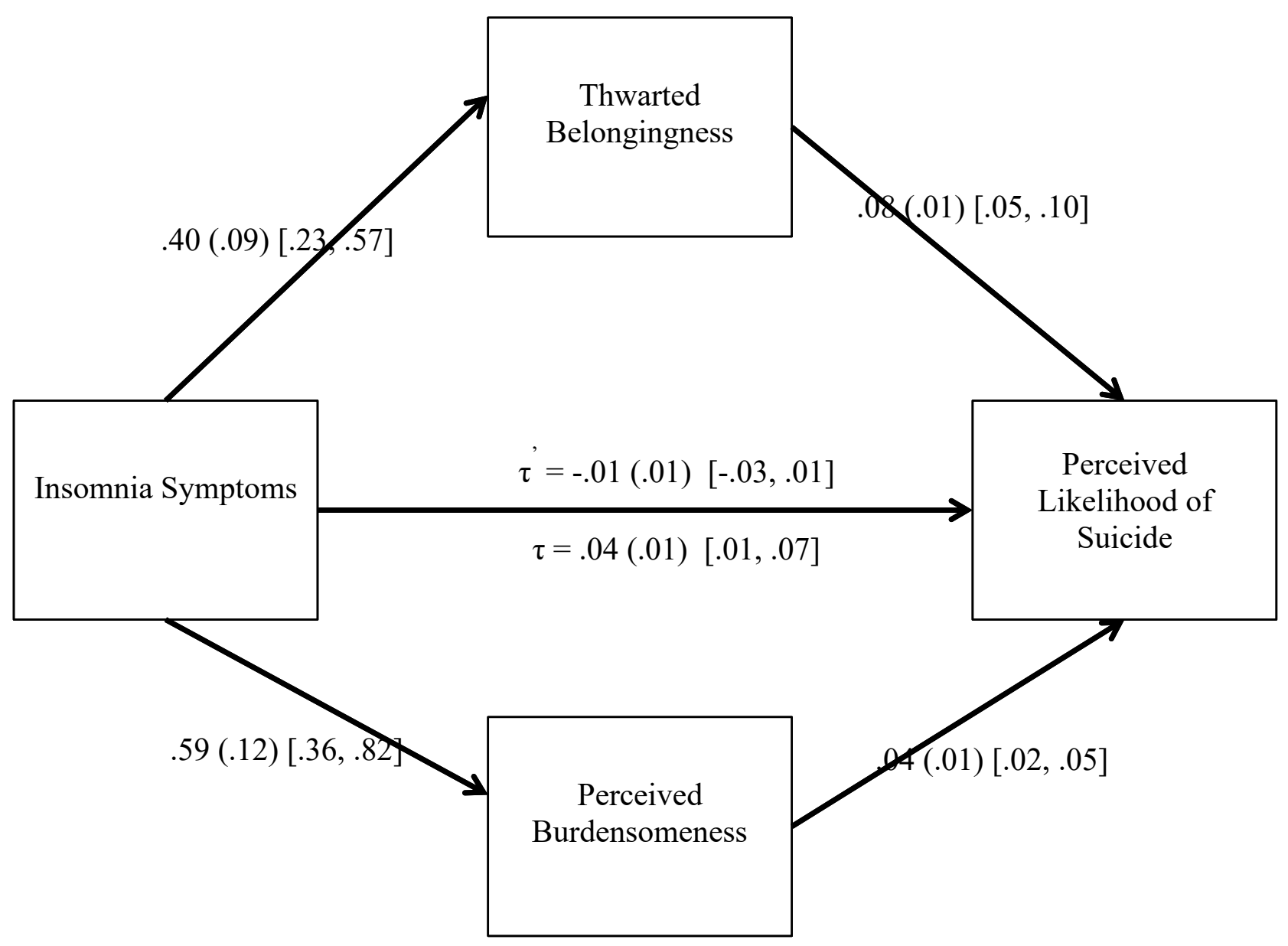

Note. Unstandardized regression coefficients, (standard errors), and [95\% confidence intervals] presented; $\tau$ ' = direct effects of independent variable on dependent variable; $\tau=$ total effects of independent variable on dependent variable. 
The results of the full model significantly predicted clinically elevated suicide risk status $\left(X^{2}(1)=64.91, p<.001\right.$, Cox \& Snell $\mathrm{R}^{2}=.29$, Nagelkerke $\mathrm{R}^{2}=.38$; Figure 3$)$. The direct effect of insomnia symptoms was not significant $(\beta=.00,95 \% \mathrm{CI}=[-.05, .05])$. Insomnia evinced an indirect relationship with suicide risk through TB ( $\beta=.05,95 \%$ CI[.19, .09]) but not $\mathrm{PB}(\beta=.02$, $95 \%$ CI[-.01,.08]).

\section{Figure 3.}

Indirect Effect Analysis of Insomnia Symptoms on Clinically Significant Suicide Risk Screening Status Through Thwarted Belongingness and Perceived Burdensomeness

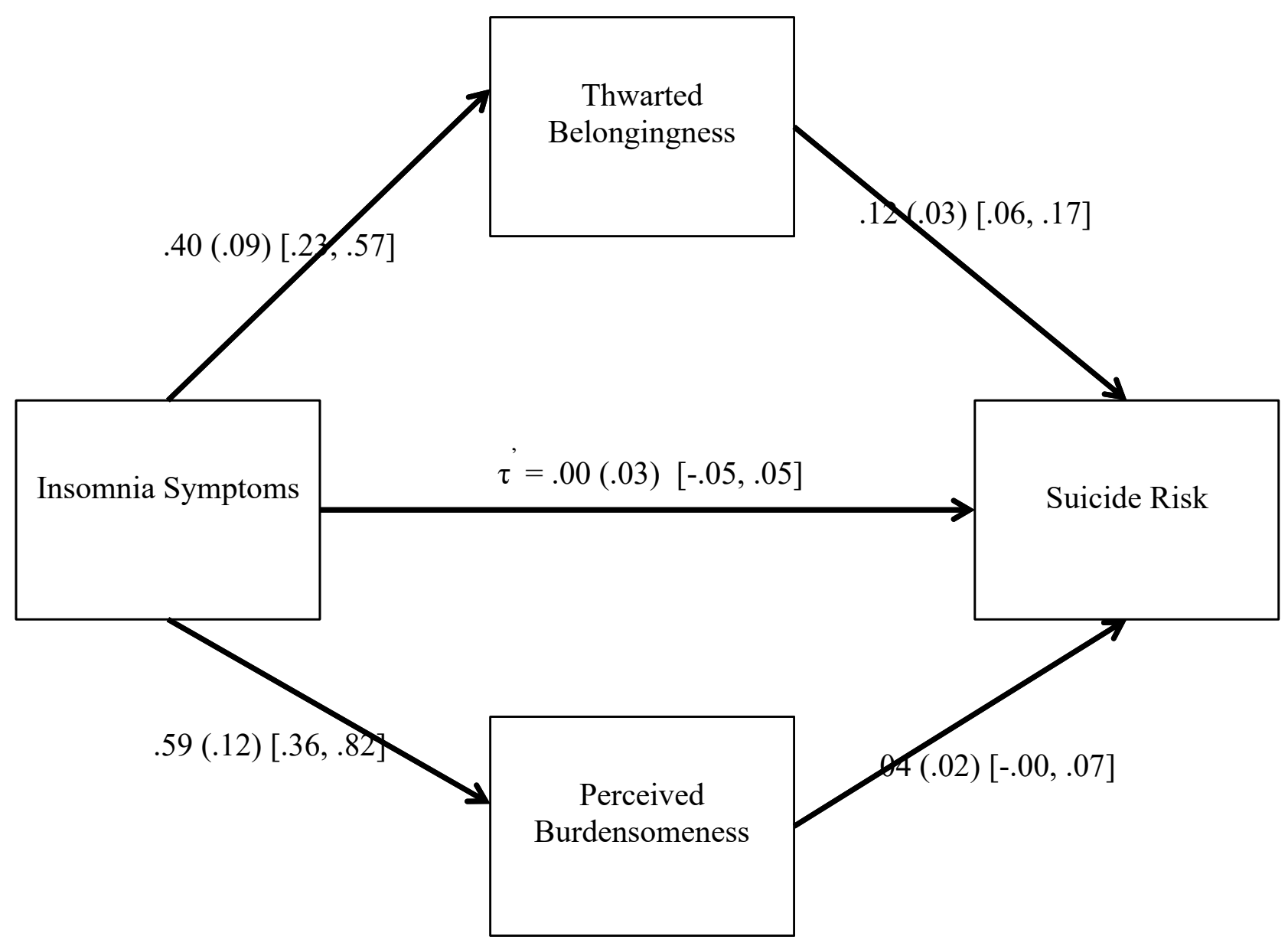

Note. Unstandardized regression coefficients, (standard errors), and [95\% confidence intervals] presented; $\tau^{\prime}=$ direct effects of independent variable on dependent variable; Suicide Risk $=$ SBQ-R clinical cut-off status dummy coded as $0=$ below cutoff and 1 $=$ above cutoff 


\section{Study 2 Method}

\subsection{Participants}

A total of 151 college students completed an online survey. Participants were generally young adults $\left(M_{\text {years }}=19.86, S D=2.67\right.$, range $\left.18-41\right)$. The majority of participants self-identified as female $(n=114,75.5 \%)$ and White $(n=120,79.5 \%)$. Table 1 contains descriptive statistics for the sample health related variables. Over half of participants qualified as elevated risk for suicide (Osman et al., 2001), whereas mean levels of insomnia were subthreshold (Morin et al., 2011).

\subsection{Measures}

4.2.1 Demographics. A demographic form was completed that included age, selfidentified gender, and race.

4.2.2 Suicidal Thoughts and Behaviors (STBs). The SBQ-R used in Study 1 was also employed in Study 2 to screen for clinically elevated suicide risk and measure perceived likelihood of future suicide.

4.2.3 Suicidal Ideation (SI). The Hopelessness Depressive Symptom QuestionnaireSuicidality Subscale (HDSQ-SS) is a four-item assessment measure of past two-week SI (Metalsky \& Joiner, 1997). Items are rated on a one to four likert-type scale with higher scores indicating increased intensity of SI. Internal consistency for the HDSQ-SS in Study 2 was good $(\alpha=.87)$. This measure replaced the SBQ-R item two as a SI outcome given it is not a single item measure and its time course is closer to the date of assessment (i.e., past two weeks compared to past-year SI).

4.2.4 Insomnia Symptoms. The ISI used in Study 1 was also employed in Study 2 to assess insomnia symptoms. Internal consistency for the ISI in Study 2 was good $(\alpha=.83)$. 
4.2.5 Interpersonal Cognitions. The INQ-15 used in Study 1 was also used in Study 2 to assess TB and PB. Internal consistency was high for both subscales in the present sample: PB ( $\alpha$ $=.95)$ and thwarted belonging $(\alpha=.92)$.

4.2.6 Interpersonal Hopelessness. The Interpersonal Hopelessness Scale is a 10-item self-report measure of perceived intractability of TB and PB (Tucker et al., 2018). Participants rate each item on a likert-type scale from one to five with higher scores representing increased interpersonal hopeless cognitions. Although the scale was derived to include five items for hopelessness about TB and five items representing intractability of perceptions of burdensomeness, previous psychometric investigations support a one factor solution and thus the total score of the measure was utilized (Mandracchia et al., 2019; Tucker et al., 2018). The internal consistency of the IHS in the current study was excellent $(\alpha=.95)$.

\subsection{Procedure}

A full description of study procedures can be found elsewhere (Tucker et al., 2018). Briefly, participants were recruited via a research participation pool of undergraduate students. SBQ-R item 1 that assesses history of STBs was completed by all students in the research pool during a pre-screen assessment at the beginning of each semester. Only participants who endorsed a history of SI or past suicidal behavior were invited to participate (SBQ-R item 1 score of 2 or above). Thus, all participants were undergraduate students, 18 years of age or older, with a history of suicidal thoughts and/or behaviors. Participants completed all study measures via an online survey platform and provided consent by clicking a labeled button upon reading a study description sheet. They were compensated with course credit for their participation. All study procedures were reviewed by the appropriate institutional review board.

\subsection{Analytical Strategy}


Similar to Study 1, missing data were imputed using multiple imputation techniques. Bivariate correlations were conducted to test simple associations and non-parametric bootstrapping procedures with the SPSS PROCESS macro was used to test multiple mediation models. Three models in total were conducted, all of which included TB, PB, and interpersonal hopelessness as parallel mediators and age and gender as covariates. The first model tested the indirect effect of insomnia symptoms on past two-week SI through the mediators. Model two tested the indirect effect of insomnia symptoms on perceived likelihood of future suicide through the mediators. Model three tested the indirect effect of insomnia symptoms on clinically significant suicide risk screening (dummy coded as $0=$ negative screen, $1=$ positive screen) through the mediators.

\section{Study 2 Results}

Missing data on any scale items was low (maximum 4.2\%). Table 2 contains bivariate associations. Insomnia displayed significant small positive correlations with suicide risk screen status, interpersonal cognitions and, interpersonal hopelessness. Unlike Study 1, insomnia was unrelated to SI $(p=.062)$ and perceived likelihood of future suicide $(p=.060)$. Interpersonal cognitions and interpersonal hopelessness displayed significant moderate positive associations with suicide outcomes.

\subsection{Parallel mediation findings}

The results of the full model significantly predicted past two-week SI $\left(R^{2}=.34, F(6\right.$, $144)=12.42, p<.001 ;$ Figure 4$)$. The direct effect of insomnia symptoms on SI was not significant $(\beta=-.00,95 \% \mathrm{CI}[-.06, .05])$. An indirect effect for insomnia symptoms on SI was observed through TB $(\beta=.03,95 \% \mathrm{CI}[.01, .06])$ and $\mathrm{PB}(\beta=.03,95 \% \mathrm{CI}[.01, .07])$ but not interpersonal hopelessness $(\beta=.01,95 \% \mathrm{CI}[-.02, .03])$. A pairwise comparison of the indirect effects 
demonstrated no statistical difference in strength between the two effects $(\beta=.01,95 \% \mathrm{CI}[-$

$.04, .05])$

Figure 4.

Indirect Effect Analysis of Insomnia Symptoms on Suicide Ideation Through Thwarted Belongingness, Perceived Burdensomeness, and Interpersonal Hopelessness

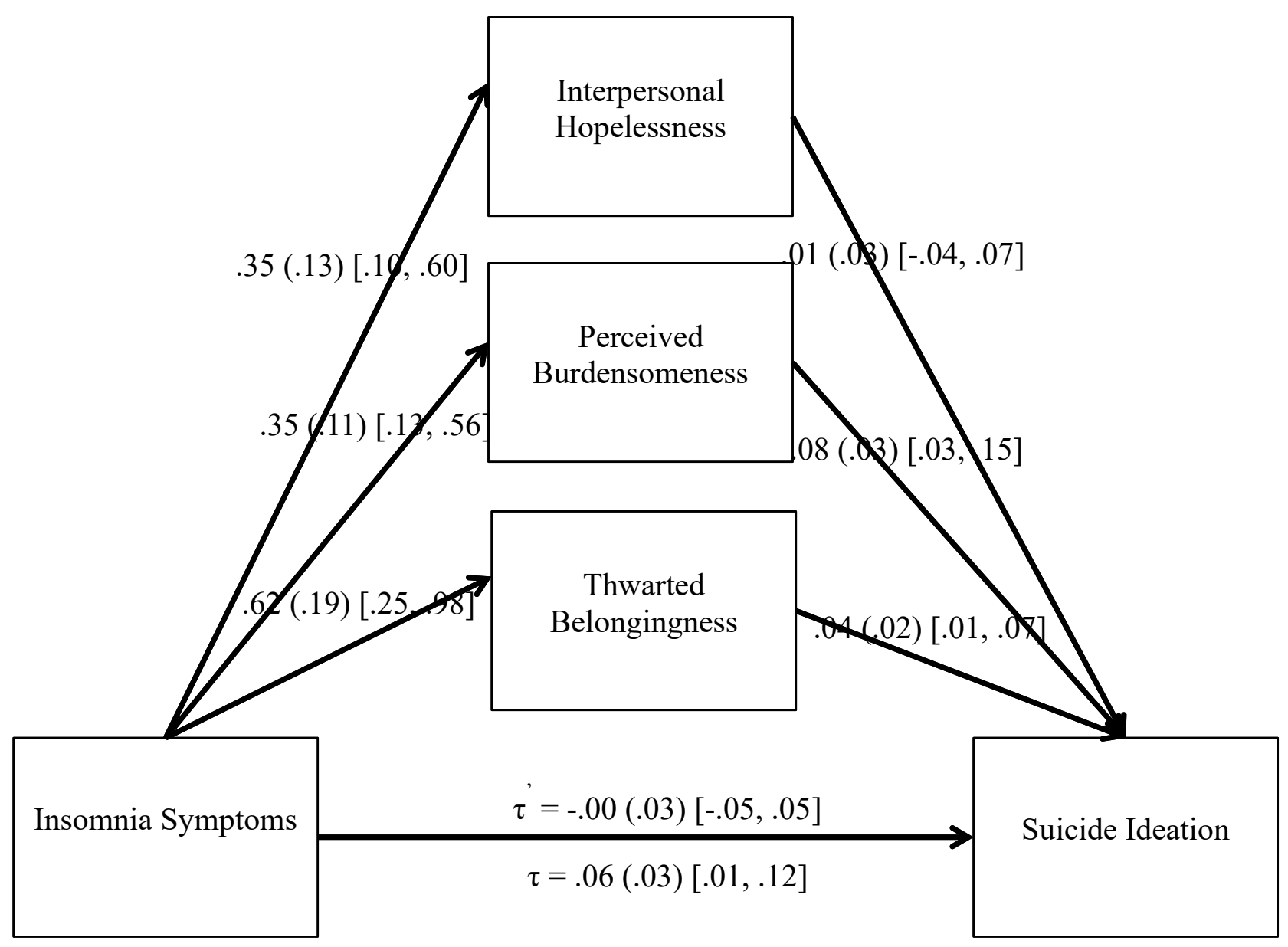

Note. Unstandardized regression coefficients, (standard errors), and [95\% confidence intervals] presented; $\tau$ ' = direct effects of independent variable on dependent variable; $\tau=$ total effects of independent variable on dependent variable; Suicide Ideation $=$ severity of suicide ideation in the past two-weeks. 
The results of the full model significantly predicted perceived likelihood of future suicide $\left(R^{2}=.29, \mathrm{~F}(6,144)=9.98, p<.001 ;\right.$ Figure 5$)$. The direct effect of insomnia symptoms on likelihood of future suicide was not significant $(\beta=.00,95 \% \mathrm{CI}[-.03, .03])$. An indirect effect for insomnia symptoms on perceived suicide risk was seen through $\mathrm{TB}(\beta=.02,95 \% \mathrm{CI}[.01, .04])$ but not PB $(\beta=.01,95 \%$ CI[-.01,.03]) or interpersonal hopelessness ( $\beta=-.00,95 \% \mathrm{CI}[-.01, .01])$.

Figure 5.

Indirect Effect Analysis of Insomnia Symptoms on Perceived Risk for Future Suicide Attempt Through Thwarted Belongingness, Perceived Burdensomeness, and Interpersonal Hopelessness

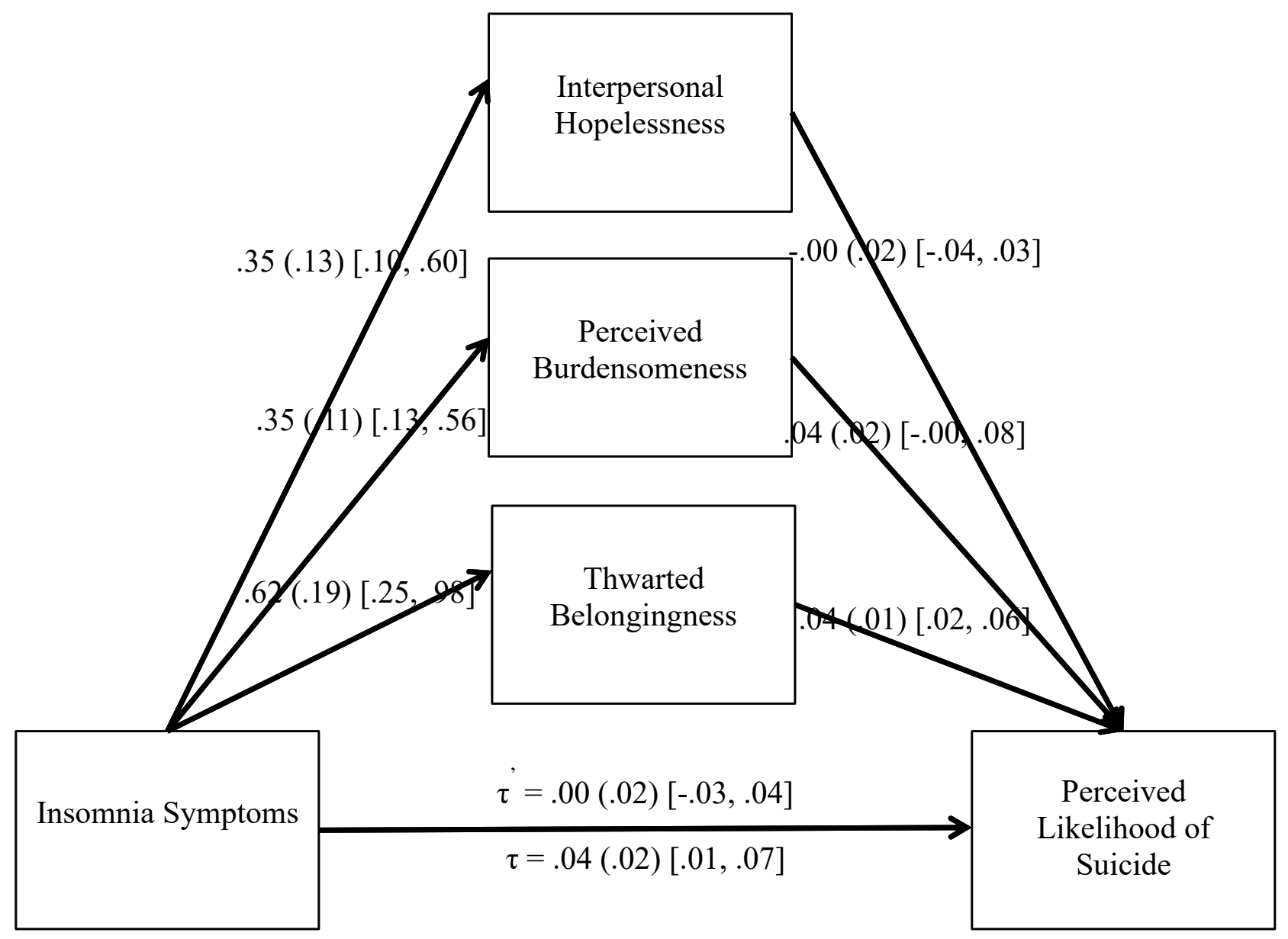

Note. Unstandardized regression coefficients, (standard errors), and [95\% confidence intervals] presented; $\tau^{\prime}=$ direct effects of independent variable on dependent variable; $\tau=$ total effects of independent variable on dependent variable. 
The results of the full model significantly predicted significant screen for suicide risk $\left(X^{2}\right.$ $(1)=35.58, p<.001$, Cox $\&$ Snell $R^{2}=.21$, Nagelkerke $R^{2}=.30$; Figure 6). The direct effect of insomnia symptoms on likelihood of future suicide was not significant $(\beta=.02,95 \% \mathrm{CI}[-$ $.05, .08])$. An indirect effect for insomnia symptoms on positive suicide risk screen was seen through TB $(\beta=.04,95 \% \mathrm{CI}[.01, .10])$ but not $\mathrm{PB}(\beta=.04,95 \% \mathrm{CI}[-.01, .12])$ or interpersonal hopelessness $(\beta=-.01,95 \% \mathrm{CI}[-.05, .07])$.

\section{Figure 6.}

Indirect Effect Analysis of Insomnia Symptoms on Clinically Significant Suicide Risk Screening Status Through Thwarted Belongingness, Perceived Burdensomeness, and Interpersonal Hopelessness

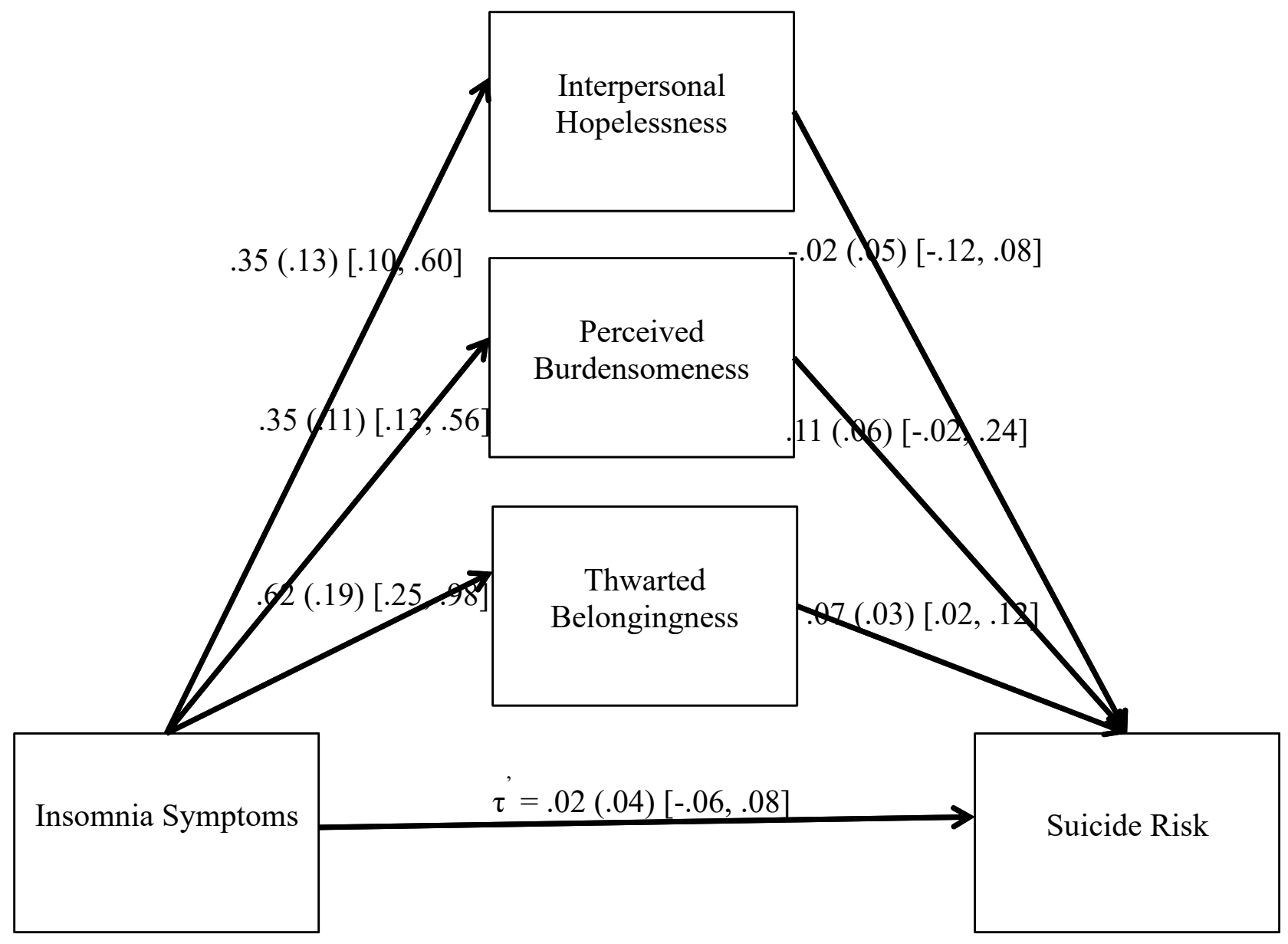

Note. Unstandardized regression coefficients, (standard errors), and [95\% confidence intervals] presented; $\tau^{\prime}=$ direct effects of independent variable on dependent variable; Suicide Risk $=$ SBQ-R clinical cut-off status dummy coded as $0=$ below cutoff and 1 $=$ above cutoff 


\section{Discussion}

The current set of studies expanded upon research demonstrating the potential mechanistic role TB plays in the relationship between insomnia symptoms and STBs (Chu et al., 2017). Continued research that explains the insomnia-suicide link is necessary (Woznica et al., 2015) and prior research has demonstrated the particular relevance of TB as an important mechanism in various military samples. However, no research has specifically investigated these relationships in a clinical sample of active duty personnel/high risk college student sample or considered the influence of interpersonal hopelessness. The current set of studies filled these important gaps in the literature.

Study 1 demonstrated the indirect effect of insomnia through TB on all three suicide outcomes: past-year SI, one's own perceived risk for suicide, and screening for clinically significant suicide risk, although insomnia evinced an indirect effect through PB for the first two outcomes well. These findings clearly replicate and extend previous work in a sample of Army recruiters demonstrating the significance of TB in understanding the insomnia and STB relationship (Hom et al., 2017). These findings were expanded upon within Study 2 through inclusion of interpersonal hopelessness as a mediator. Again, an indirect effect of insomnia through TB on the three indicators of suicide risk was observed. Insomnia symptoms are related to increased emotion dysregulation difficulties (Jansson-Fröjmark et al., 2016) and difficulty managing stressors (Minkel et al., 2012), all of which may increase likelihood of perceived social isolation and burdensomeness. In addition, the emotion regulation difficulties coupled with insomnia symptoms such as irritability may also decrease day-to-day positive social interactions and thus one's social connectedness as hypothesized by Chu and colleagues (2017). This constellation of sleep, mood, and social difficulties may over time be experienced as so 
aversive that suicide is seen as the only viable option of coping with them. This hypothesis is supported by research demonstrating reduced problem-solving ability and broader executive functioning concerns in both those with a history of STBs (Jollant et al., 2011) as well as those experiencing insomnia (Warble-Pinkston, Slavish, \& Taylor, 2019).

Contrary to hypotheses, an indirect effect of insomnia on the aforementioned suicide risk outcomes through interpersonal hopelessness was not observed. Previous research demonstrates that interpersonal hopelessness, but not general hopelessness, moderates the relationship between the interaction of TB and PB and severity of suicidal thinking (Tucker et al., 2018). Although interpersonal hopelessness appears to play an important role in vulnerability for suicidal thinking even when other related risk factors are present, it may not explain why insomnia is related to SI or individual perceived risk for suicide. Additionally, the IPTS posits that interpersonal hopelessness plays an important role in the development of suicidal desire and not necessarily passive SI (i.e., desire to be dead) or even perceived risk for a suicide attempt (Van Orden et al., 2010; Baca-Garcia et al., 2011). Of note, the measure of SI used in Study 2 includes items that assess planning for suicide and inability to control suicidal impulses and thus is not a perfect tool for assessing suicidal desire. Future research should seek to replicate these results with this concern in mind.

These study results have potentially meaningful implications for clinical care. First, results indicate that the assessment of TB in the context of clinical care for insomnia may be important for understanding patient vulnerability for suicidal thinking, especially in light of evidence supporting the use of the INQ within clinical assessment for and monitoring of STBs within civilian and military samples alike (Gutierrez et al., 2016; Mitchell et al., 2019). When elevated, a brief intervention that reduces TB, such as the web-based LEAP intervention (Hill \& 
Pettit, 2016), may be a useful addition to treatment. Briefly, LEAP is a selective preventative intervention that specifically identifies and targets feelings of PB toward ultimate reduction of risk for suicidal thoughts and has proven effective at reducing feelings of PB at post-treatment and follow-up when compared to a psychoeducation intervention (Hill \& Pettit, 2016). This adjunctive clinical treatment approach may also be important for those who present for primary suicide-related concerns. Suicide-specific interventions may benefit from the assessment and treatment of sleep-related concerns such as insomnia symptoms in tandem with other issues that increase suicide risk. In corroboration with this point, Brief Cognitive Behavioral Therapy (BCBT; Bryan \& Rudd, 2018) includes CBT strategies at the core of CBT for Insomnia (CBTi; Bennett, 2020) to help reduce insomnia concerns. As the field of suicide-specific care continues to grow (Jobes, 2017), strategies to reduce sleep-related concerns should be considered.

The absence of an indirect effect of insomnia on suicide-related outcomes through interpersonal hopelessness also has meaningful implications. Although this newly measured construct was positively related to insomnia symptoms, the process in which insomnia increases vulnerability for suicidal thoughts may not be through hopelessness about unmet interpersonal needs, but specifically through TB across populations. That is, for both college students and active-duty military service members, TB explains the relationship between insomnia and suicidal thoughts and behaviors, indicating a potentially more generalizable trend. Notably, while PB and interpersonal hopelessness appear to be more strongly related to suicidal thinking compared to TB in isolation (Tucker et al., 2018), insomnia may not confer the same level of vulnerability to these concerns as it does TB. Again, this result indicates the importance of assessing and reducing TB specifically in the context of primary concerns with insomnia. 
Further, it is worth noting the indirect effect of insomnia on suicide related outcomes through PB in the active duty military sample. Indeed, it is possible that the impact of insomnia in active duty service members may be more pronounced leading to PB. As many experts note, the collective nature of the military can offer protective benefits but can also become a detriment if a given service member is ostracized from the collective for one reason or another (e.g., detriment to force efficacy) (Bryan et al., 2012). It may be that sleep-related concerns are perceived as impacting one's performance in the broader unit, potentially leading to PB and thus suicidal thinking. Nevertheless, this finding should be interpreted with caution in light of the small effect sizes obtained in the indirect effect models related to PB, insomnia, and suicide outcomes.

\subsection{Limitations}

Sample limitations in both studies should be considered when interpreting results. First, both studies included participants that predominantly self-identified as White. As such, results may not generalize to racially diverse samples. Generalizability of Study 1 is also limited given the sample was predominantly recruited from a substance abuse facility. Given research that indicates insomnia concerns prospectively confer risk for problematic alcohol use (Short et al., 2019) and alcohol use confers risk for TB, PB and thus SI (Gallyer et al., 2018), Study 1 results could be conflated by high rates of alcohol (and other substance) abuse and may not generalize to other treatment-seeking populations. Methodological limitations across the two studies should also be considered. First, both studies are cross-sectional and thus cannot produce meaningful causal or temporal assertions about the relationship between insomnia, unmet interpersonal needs, and suicide-related outcomes. Further, although the SBQ-R is a well validated, commonly used measure of suicide risk, using single items from the measure is not ideal as single item 
assessments may overestimate true prevalence of suicidal-related outcomes (Hom, Joiner, \& Bernert, 2016). The utilization of item two of the SBQ-R in Study 1 as the primary indicator of SI is not ideal as participants rated frequency of SI in the past year whereas insomnia symptoms were rated in the past two-weeks. This concern is not relevant for Study 2 however as the time course of SI and insomnia symptoms were rated over the past two-weeks thus improving on this methodological concern in Study 1. Likewise, the assessment of insomnia symptoms is not ideal. Future research should utilize semi-structured clinical interviews to better understand the presence of and distressed experienced by insomnia symptoms. This methodological improvement would also allow for the formal diagnosis of insomnia and its relationship with unmet interpersonal needs and suicidal thoughts and perceived risk for suicide.

\section{Conclusions}

The current set of studies add critical incremental understanding to the link between insomnia and STBs. The available research (Britton et al., 2019; Chu et al., 2017; Hom et al., 2017), coupled with the current results, appears to indicate that TB and PB explain the relationship in question, although $\mathrm{PB}$ emerged as a mechanism of action in only the active-duty sample representing a potential population specific mechanism worthy of future research. Further, although the results of Study 1 preclude causal conclusions, the observation at the heart of this study may now include active-duty military service members, enhancing the generalizability of the explanatory power of TB in the insomnia-STB link, if future work replicates the finding in prospective samples. Thus, future empirical work that explores the question of why TB consistently explains the relationship between insomnia and STBs is recommended. 


\section{References}

Baca-Garcia, E., Perez-Rodriguez, M. M., Oquendo, M. A., Keyes, K. M., Hasin, D. S., Grant, B. F., Blanco, C. (2011). Estimating risk for suicide attempt: Are we asking the right questions?: Passive suicidal ideation as a marker for suicidal behavior. Journal of Affective Disorders, 134(1-3), 327 - 332.

Bennett, D. (2020). Cognitive-Behavioral Therapy for Insomnia (CBT-I). In Sleep Medicine and Mental Health (pp. 47-66). Springer, Cham.

Bernert, R. A. \& Joiner, T. E. (2007). Sleep disturbances and suicide risk: a review of the literature. Neuropsychiatric Disease and Treatment, 3, 735-743.

Bishop, T. M., Crean, H. F., Hoff, R. A., \& Pigeon, W. R. (2019). Suicidal ideation among recently returned veterans and its relationship to insomnia and depression. Psychiatry Research, 276, 250-261.

Blasco-Fontecilla, H., Alegria, A. A., Lopez-Castroman, J., Legido-Gil, T., Saiz-Ruiz, J, de Leon, J., Baca-Garcia, E. (2011). Short self-reported sleep duration and suicidal behavior: A cross-sectional study. Journal of Affective Disorders, 133, 239-246.

Britton, P. C., McKinney, J. M., Bishop, T. M., Pigeon, W. R., \& Hirsch, J. K. (2019). Insomnia and risk for suicidal behavior: A test of a mechanistic transdiagnostic model in veterans. Journal of Affective Disorders, 245, 412-418.

Bryan, C. J., Jennings, K. W., Jobes, D. A., \& Bradley, J. C. (2012). Understanding and Preventing Military Suicide. Archives of Suicide Research, 16, 95 - 110.

Bryan, C. J. \& Rudd, M. D. (2018). Brief Cognitive-Behavioral Therapy for Suicide Prevention. New York, NY: The Guilford Press.

Bos, S. C. \& Macedo, A. F. (2019). Literature review on Insomnia (2010-2016). Biological 
Rhythm Research, 50(1), 94-163.

Chattu, V. K., Manzar, M. D., Kumary, S., Burman, D., Spence, D. W., \& Pandi-Perumal, S. R. (2019, March). The global problem of insufficient sleep and its serious public health implications. In Healthcare (Vol. 7, No. 1, p. 1). Multidisciplinary Digital Publishing Institute.

Chu, C., Hom, M. A., Rogers, M. L., Stanley, I. H., Ringer-Moberg, F. B., Podlogar, M. C., Hirsch, J. K., \& Joiner, T. E. (2017). Insomnia and suicide-related behaviors: A multistudy investigation of thwarted belongingness as a distinct explanatory factor. Journal of Affective Disorders, 208, 153-167.

Cramer, R. J., Mandracchia, J., Gemberling, T. M., Holley S. R., Wright, S., Moody, K., \& Nobles, M.R. (2017). Can Need for Affect and sexuality differentiate suicide risk in three community samples? Journal of Social and Clinical Psychology, 36, 704-722.

Cramer, R. J., Rasmussen, S., Webber. W. B., Sime, V. L., Haile, C., McFadden, C., \& McManus, M. C.(2019). Preferences in Information Processing and suicide: Results from a young adult health survey in the United Kingdom. International Journal of Social Psychiatry, 65, 46-55.

Cunningham, C. A., Cramer, R. J., Cacace, S., Franks, M., \& Demarais, S. L. (2020). The Coping Self Efficacy Scale: Psychometric properties in an outpatient sample of active duty military personnel. Military Psychology, 32, 261-272.

Crosby, A., Gfroerer, J., Han, B., Ortega, L., \& Parks, S. E. (2011). Suicidal thoughts and behaviors among adults aged $>18$ Years--United States, 2008-2009. Retrieved from: https://stacks.cdc.gov/view/cdc/6021 
Department of the Navy (2016). Operational Stress Control Program. Office of the Chief of Naval Operations, OPNAV INSTRUCTION 6520.1A, 14 Jun 2016.

Deshong, H. L., \& Tucker, R. P. (2019). Borderline personality disorder traits and suicide risk: The mediating role of insomnia and nightmares. Journal of Affective Disorders, 244, 8591.

Ellis, J. G., Perlis, M. L., Neale, L. F., Espie, C. A., \& Bastien, C. H. (2012). The natural history of insomnia: focus on prevalence and incidence of acute insomnia. Journal of Psychiatric Research, 46(10), 1278-1285.

Enders, C. K. (2017). Multiple imputation as a flexible tool for missing data handling in clinical research. Behaviour Research and Therapy, 98, 4-18

Ferentinos, P., Porichi, E., Christodoulou, C., Dikeos, D., Papageorgiou, C., Douzenis, A. (2016). Sleep disturbance as a proximal predictor of suicidal intent in recently hospitalized attempts. Journal of Affective Disorders, 19, 1-7.

Gallyer, A. J., Dougherty, S. P., Gai, A. R., Stanley, I. H., Hom, M. A., Rogers, M. L., ... \& Joiner, T. E. (2018). Problematic alcohol use and suicidal ideation among firefighters: A multi-study investigation of the explanatory roles of perceived burdensomeness and thwarted belongingness. Journal of Affective Disorders, 238, 281-288.

Gutierrez, P. M., Joiner, T., Hanson, J., Avery, K., Fender, A., Harrison, T., ... \& Rogers, M. L. (2020). Clinical utility of suicide behavior and ideation measures: Implications for military suicide risk assessment. Psychological Assessment. Advanced online publication. Gutierrez, P. M., Pease, J., Matarazzo, B. B., Monteith, L. L., Hernandez, T., Osman, A. (2016). Evaluating the psychometric properties of the Interpersonal Needs Questionnaire and the 
Acquired Capability for Suicide Scale in military veterans. Psychological Assessment, 28(12), $1684-1694$.

Hayes, A. F. (2018). Introduction to mediation, moderation, and conditional process analysis: A regression-based approach. Guilford Press.

Hill, R. M., \& Pettit, J. W. (2019). Pilot randomized controlled trial of LEAP: A selective preventive intervention to reduce adolescents' perceived burdensomeness. Journal of Clinical Child \& Adolescent Psychology, 48(sup1), S45-S56.

Hom, M. A., Joiner Jr, T. E., \& Bernert, R. A. (2016). Limitations of a single-item assessment of suicide attempt history: Implications for standardized suicide risk assessment. Psychological Assessment, 28(8), 1026.

Jansson-Fröjmark, M., Norell-Clarke, A., \& Linton, S. J. (2016). The role of emotion dysregulation in insomnia: Longitudinal findings from a large community sample. British Journal of Health Psychology, 21(1), 93-113.

Jobes, D. A. (2017). Clinical assessment and treatment of suicidal risk: A critique of contemporary care and CAMS as a possible remedy. Practice Innovations, 2(4), 207.

Joiner, T. (2007). Why people die by suicide. Harvard University Press.

Jollant, F., Lawrence, N. L., Olié, E., Guillaume, S., \& Courtet, P. (2011). The suicidal mind and brain: a review of neuropsychological and neuroimaging studies. The World Journal of Biological Psychiatry, 12(5), 319-339.

Kleiman, E. M., \& Anestis, M. D. (2015). Introduction to the special issue: Recent advances in suicide research: Mediators and moderators of risk and resilience. International Journal of Cognitive Therapy, 8(2), 95-98.

Klingaman, E. A., Brownlow, J. A., Boland, E. M., Mosti, C., \& Gehrman, P. R. (2018). 
Prevalence, predictors and correlates of insomnia in US army soldiers. Journal of Sleep Research, 27(3), e12612.

Krakow, B., Ribeiro, J. D., Ulibarri, V. A., Krakow, J., \& Joiner Jr, T. E. (2011). Sleep disturbances and suicidal ideation in sleep medical center patients. Journal of Affective Disorders, 131(1-3), 422-427.

Kupfer, D. J., \& Reynolds, C. F. (1997). Management of insomnia. New England Journal of Medicine, 336(5), 341-346.

Lamberg, L. (1997). World Health Organization targets insomnia. JAMA, 278(20), 1652.

Littlewood, D., Kyle, S. D., Pratt, D., Peters, S., \& Gooding, P. (2017). Examining the role of psychological factors in the relationship between sleep problems and suicide. Clinical Psychology Review, 54, 1-16.

Lund, E. M., Nadorff, M. R., Galbraith, K., \& Thomas, K. B. (2019). Comparing the internal consistency, overall scores, and response patterns on the Suicidal Behavior Questionnaire-Revised in people with and without disabilities. Rehabilitation Counseling Bulletin, 62(2), 108-120. 1-3, 422-427.

Ma, J., Batterham, P. J., Calear, A. L., \& Han, J. (2016). A systematic review of the predictions of the Interpersonal-Psychological Theory of Suicidal Behavior. Clinical Psychology Review, 46, 34-45.

Malik, S., Kanwar, A., Sim, L. A., Prokop, L. J., Wang, Z., Benkhadra, K., \& Murad, M. H. (2014). The association between sleep disturbances and suicidal behaviors in patients with psychiatric diagnoses: a systematic review and meta-analysis. Systematic Reviews, 3(1), 1-9. 
Mandracchia, J. T., Sunderland, M. N., \& To, Y. M. (2019). Evaluating the role of interpersonal hopelessness in the interpersonal theory of suicide. Death Studies, 1-5.

McGlinchey, E. L., Courtney-Seidler, E. A., German, M., \& Miller, A. L. (2017). The role of sleep disturbance in suicidal and nonsuicidal self-injurious behavior among adolescents. Suicide and Life-Threatening Behavior, 47(1), 103-111.

Metalsky, G. I. \& Joiner, T. E., Jr. (1997). The Hopelessness Depression Symptom Questionnaire. Cognitive Therapy and Research, 21, 359-384.

Minkel, J. D., Banks, S., Htaik, O., Moreta, M. C., Jones, C. W., McGlinchey, E. L., ... \& Dinges, D. F. (2012). Sleep deprivation and stressors: evidence for elevated negative affect in response to mild stressors when sleep deprived. Emotion, 12(5), 1015.

Mitchell, S. M., Brown, S. L., Roush, J. F., Tucker, R. P., Cukrowicz, K. C., \& Joiner, T. E. (2019). The Interpersonal Needs Questionnaire: Statistical considerations for improved clinical application. Assessment, 27(3), $621-637$.

Morin, C. M. (1993). Insomnia: psychological assessment and management. Guilford Press.

Morin C. M., Belleville, G., Bélanger, L., \& Ivers, H. (2011). The insomnia severity index: psychometric indicators to detect insomnia cases and evaluate treatment response. SLEEP, 34(5), 601-608.

Osman, A., Bagge, C. L., Gutierrez, P. M., Konick, L. C., Kopper, B. A., \& Barrios, F. X. (2001). The Suicidal Behaviors Questionnaire-Revised (SBQ-R): Validation with clinical and non-clinical samples. Assessment, 8, 443-454.

Park, W., Kwang, I. Y., Kim, H. (2019). Insufficient sleep and suicidal ideation: a survey of 12,046 female adolescents. Journal of Affective Disorders, 53, 65-69. 
Perlis, M. L., Vargas, I., Ellis, J. G., Grandner, M. A., Morales, K. H., Gencarelli, A...Thase, M. E. (2020). The Natural History of Insomnia: the incidence of acute insomnia and subsequent progression to chronic insomnia or recovery in good sleeper subjects. Sleep, 43(6), zsz299.

Ribeiro, J. D., Pease, J. L., Gutierrez, P. M., Silva, C., Bernert, R. A., Rudd, M. D., Joiner Jr., T. E. (2012). Sleep problems outperform depression and hopelessness as cross-sectional and longitudinal predictors of suicidal ideation and behavior in young adults in the military. Journal of Affective Disorders, 136. 743-750.

Rucker, D. D., Preacher, K. J., Tormala, Z. L., \& Petty, R. E. (2011). Mediation analysis in social psychology: Current practices and new recommendations. Social and Personality Psychology Compass, 5/6, 359-371.

Short, N. A., Allan, N. P., Oglesby, M. E., Moradi, S., Schmidt, N. B., \& Stecker, T. (2019). Prospective associations between insomnia symptoms and alcohol use problems among former and current military service personnel. Drug and Alcohol Dependence, 199, 3541.

Stanley, I. H., Hom, M. A., Rogers, M. L., Hagan, C. R., \& Joiner, T. E. (2016). Understanding suicide among older adults: A review of psychological and sociological theories of suicide. Aging \& Mental Health, 20(2), 113-122.

Stanley, I. H., Joiner, T. E., \& Bryan, C. J. (2017). Mild traumatic brain injury and suicide risk among a clinical sample of deployed military personnel: Evidence for a serial mediation model of anger and depression. Journal of Psychiatric Research, 84, 161-168. 
Tucker, R. P., Hagan, C. R., Hill, R. M., Slish, M. L., Bagge, C. L., Joiner Jr, T. E., \& Wingate, L. R. (2018). Empirical extension of the interpersonal theory of suicide: Investigating the role of interpersonal hopelessness. Psychiatry Research, 259, 427-432.

Tae, H., Jeong, B. R., Chae, J., (2019). Sleep problems as a risk factor for suicide: Are certain specific sleep domains associated with increased suicide risk? Journal of Affective Disorders, 252, 182-189.

Van Orden, K. A., Cukrowicz, K. C., Witte, T. K., \& Joiner, T. E. (2012). Thwarted belongingness and perceived burdensomeness: Construct validity and psychometric properties of the Interpersonal Needs Questionnaire. Psychological Assessment, 24(1), $197-215$.

Van Orden, K. A., Witte, T. K., Cukrowicz, K. C., Braithwaite, S. R., Selby, E. A., \& Joiner, T. E. Jr, (2010). The interpersonal theory of suicide. Psychological Review, 117(2), 575600.

Wardle-Pinkston, S., Slavish, D. C., \& Taylor, D. J. (2019). Insomnia and cognitive performance: A systematic review and meta-analysis. Sleep Medicine Reviews, 48, 101205.

Winer, E. S., Drapeau, C. W., Veilleux, J. C., \& Nadorff, M. R. (2016). The association between anhedonia, suicidal ideation, and suicide attempts in a large student sample. Archives of Suicide Research, 20(2), 265-272.

Woosley, J. A., Lichstein, K. L., Taylor, D. J., Riedel, B. W., \& Bush, A. J. (2014). Hopelessness mediates the relation between insomnia and suicidal ideation. Journal of Clinical Sleep Medicine, 10(11), 1223-1230. 
Woznica, A. A., Carney, C. E., Kuo, J. R., Moss, T. G. (2015). The insomnia and suicide link: toward an enhanced understanding of this relationship. Sleep Medicine Reviews, 22, 3746. 
Table 1. Primary Descriptive Statistics for Clinical Variables by Sample

\begin{tabular}{|c|c|c|}
\hline \multirow[t]{3}{*}{ Variable } & Military Sample & High Risk College Student Sample \\
\hline & (Study 1) & (Study 2) \\
\hline & $M(S D)$ & $M(S D)$ \\
\hline Insomnia & $10.56(7.26)$ & $10.61(5.55)$ \\
\hline Perceived Burdensomeness & $13.47(9.02)$ & $11.46(7.60)$ \\
\hline Thwarted Belonging & $29.86(12.67)$ & $26.24(13.07)$ \\
\hline Interpersonal Hopelessness & - & $16.29(8.73)$ \\
\hline 12-month Suicidal Ideation & $2.46(1.48)$ & $2.86(1.46)$ \\
\hline Past 2-week Suicidal Ideation & - & $1.95(2.11)$ \\
\hline \multirow[t]{2}{*}{ Likelihood of Future Suicide Attempt } & $1.39(1.54)$ & $1.25(1.28)$ \\
\hline & $\mathrm{N}(\%)$ & $\mathrm{N}(\%)$ \\
\hline \multicolumn{3}{|l|}{ Suicide Risk } \\
\hline None & $104(52.0 \%)$ & $47(31.1)$ \\
\hline Elevated Risk & $96(48.0 \%)$ & $104(68.9)$ \\
\hline
\end{tabular}


Table 2. Bivariate Correlations of Primary Variables by Study Sample

\begin{tabular}{|c|c|c|c|c|c|c|c|}
\hline Variable & 1 & 2 & 3 & 4 & 5 & 6 & 7 \\
\hline 1. Suicidal Ideation ${ }^{\dagger}$ & - & $.64^{*}$ & $.74^{*}$ & $.16 * * *$ & $.56 *$ & $.45^{*}$ & - \\
\hline 2. Likelihood of Future SA & $.46^{*}$ & - & $.71 *$ & $.20 * *$ & $.63 *$ & $.57 *$ & - \\
\hline 3. Suicide Risk & $.49 *$ & $.49 *$ & - & $.18 * *$ & $.50 *$ & $.43 *$ & - \\
\hline 4. Insomnia & .15 & .15 & $.16 * * *$ & - & $.31 *$ & $.32 *$ & - \\
\hline 5. Thwarted Belonging & $.51 *$ & $.51 *$ & $.42 *$ & $.26 * *$ & - & $.63 *$ & - \\
\hline 6. Perceived Burdensomeness & $.54^{*}$ & $.46^{*}$ & $.35 *$ & $.25 * *$ & $.66^{*}$ & - & - \\
\hline 7. Interpersonal Hopelessness & $.49 *$ & $.42 *$ & $.31 *$ & $.22 * *$ & $.69 *$ & $.79 *$ & - \\
\hline
\end{tabular}

Notes: $* p \leq .001, * * p \leq .01, * * * p<.05,{ }^{\dagger}$ Study 1 suicidal ideation rated in the past year but Study 2 suicidal ideation rated in the past two-weeks. Statistics above the diagonal = study 1 (Military sample); Statistics below the diagonal $=$ study $2($ high risk college student sample $) ;$ Suicide Risk $=\mathrm{SBQ}-\mathrm{R}$ clinical risk $(0=$ no risk, $1=$ elevated risk). $\mathrm{SA}=$ suicide attempt. 